\title{
Propriedades funcionais de hemoglobina e sangue completo em um mamífero aquático, o peixe-boi amazônico (Trichechus inunguis) (*)
}

\author{
Martha Farmer ('); Roy E. Weber $\left({ }^{2}\right)$; Joseph Bonaven tura ('); Robin C. Best ${ }^{(3)}$; Daryl Paul Domining ( ${ }^{(3)}$
}

Resumo

O hematócrito $(43 \%)$ e a capacidade de ligação de $\mathrm{O}$ $(18,8 \mathrm{ml} \mathrm{O} / 100 \mathrm{ml}$. sangue $)$ de Trichechus inunguis são baixos ao serem comparados com outros mamífe. ros mergulhadores, mas são similares aos de mamíferos terrestres. Hb fracionada de peixe-boi é similar a $\mathrm{HbA}$ humana na sensitividade a $\mathrm{pH}, 2,3$ - difosfoglicerato e $\mathrm{CO}_{2}$, porém é menos sensitiva à temperatura $\mathrm{e}$ tem maior tendência a dissociar-se em dímeros. As notáveis curvas de Hill não exibem cooperatividade خे saturação de oxigênio inferior a $30 \%$ indicando um estado " $T$ " (ou estado de baixa afinidade) altamente estabilizado; tais curvas de Hill assimétricas ao serem tomadas em conta junto com a cinética bifásica de ligação de $\mathrm{O}_{2}$, poderão indicar heterogeneidade de cadeia. A dependência de $\mathrm{pH}$ de ligação de oxigênio pela hemoglobina em estado " $T$ " aparente como visto nas curvas de Hill, é aumentada por 2.3 - difosfoglicerato, porém eliminada por $\mathrm{CO}_{2}$.

\section{INTRODUÇÃo}

Os manatis, vulgarmente chamados peixebois, são mamíferos da ordem Sirenia, porém exibindo adaptações anatômicas e fisiológicas diferentes dos Cetáceos e Pinnipedios. De fato, os Sirênios não estão aparentados com nenhum destes dois grupos, mas parecem ter evoluído com os proboscídeos (elefantes) e hyracoideos a partir de um ancestral comum (Romer, 1965). Os Sirênios vivos estão repre. sentados por só dois gêneros, Dugong e Trichechus. Com a recente extinção do peixe-boi de Seller (Hydrodamalis gigas) do Pacítico do Norte, os Sirênios remanescentes estão restritos a certas águas costeiras rasas ou a rios dos trópicos e subtrópicos. Em contraste com outros mamíferos mergulhadores, são totalmente hervíboros, alimentando-se principalmente de plantas aquáticas vasculares.
Scholander \& Irving (1941) compararam a respiraçäo e capacidade de mergulho do manati da Florida, Trichechus manatus latirostris, com a de outros mamíferos mergulhadores. Dados os hábitos alimentares do manati, seus mergulhos são geralmente rasos ao serem comparados com os de baleias e focas. Além disto, os manatis tendem a ser vagarosos ainda que capazes de nadar rapidamente durante curtos períodos. De interesse especial a este estudo, é a capacidade de $\mathrm{O}_{2}$ relativamente baixa do sangue $\left(17,2 \mathrm{ml} . \mathrm{O}_{2} / 100 \mathrm{ml}\right.$. sangue $)$ reportada para Trichechus manatus latirostris e a carência relativa de mioglobina muscular comparada com outros mamiferos mergulhadores (Blessing, 1972; Scholander \& Irving, 1941). Elsner (1968) encontrou valores de $\mathrm{O}_{2}$ similarmente baixos para o "dugong". Além destas características de baixo teor de mioglobina e baixa capacidade de $\mathrm{O}_{2}$, o volume de sangue do manati não representa além de $8 \%$ do peso corporal (Scholander \& Irving, 1941; Prosser, 1973) .

O peixe-boi amazônico tem-se tornado cada vez mais escasso e está atualmente protegido pelo governo brasileiro. Está sendo feito um esforço para determinar os requerimentos ecológicos da espécie para poder evitar sua extinção. A fisiologia, nutrição e comportamento estão sendo estudados por três de nós do INPA $\left({ }^{4}\right)$ (R.C.B., D.E. e D.M.) e os dados apresentados neste trabalho fazem parte de um estudo a longo prazo da fisiologia respiratória do manati. O presente trabalho tem por objetivo estudar as propriedades funcionais de soluçōes de hemoglobina que têm sido destituída de cofatores que modulam a afinidade de oxigê-

$\left({ }^{*}\right)$ - Versåo origlnal inglesa publicada em Comp. Biochem. Physiol vol. 62 A (1). 1979.

(1) - Duke University Marine Laboratory. Beaufort, North Carolina, 28516, E.E.U.U.

(2) - Department of Zoology, University of Aarhus, DK-800, Aarhus C Dinamarca.

(3) - Instituto Nacional de Pesquisas da Amazônia, Brasil.

(4) - As abreviaçōes usadas sāo: INPA, Instituto Nacional de Pesquisas da Amazônia: DPG, 2.3 - difosfoglicera-

- to; ATP, Adenosina trifosfato; NTP, nucleosidotrifosfato; IHP, Inositol hexafosfato;. HbA, hemoglobina hum ־na de adulto; bis-Tris, 2,2 - bis (hidroximentil - 2.2'22". - nitriloetanol; EDTA, ácido etilenodiamino tetraacético; I, força iônica; $\mathrm{P}_{50}$, tensão de oxigênio em $\mathrm{mm}$ de mercúrio (torr) que resulta em meia saturação de hemogı́obina com oxigênilo; $\mathbf{n}$, pendente da curva de Hill de ligação de oxigênio calculada a $\mathrm{P}_{50}$. 
nio in vivo. Os efeitos de $\mathrm{pH}$, temperatura, DPG e $\mathrm{CO}_{2}$ sobre a afinidade de oxigênio da hemoglobina foram estudados usando métodos de equilíbrio e cinética.

\section{MATERIAIS E MÉTODOS}

Duas fêmeas novas do INPA, uma pesando 14 e a outra $19,5 \mathrm{~kg}$, foram levadas a bordo do R/V "Alpha Helix". As duas foram capturadas na época seca de 1976 no Paraná do Autaz-Açu, perto da desembocadura do rio Madeira. Ainda que as duas fêmeas tivessem menos de 1 ano de idade na época deste estudo, a diferença de tamanho sugere que não são irmãs.

Uma pequena amostra de sangue foi tirada de cada animal para exame eletroforético. As eletroforeses de gel de disco de acrilamida (gels 7,5\%) foram realizadas segundo o método descrito por Fyhn et. al., (1978) .

Para os estudos funcionais foram tirados $5 \mathrm{ml}$ de sangue da nadadeira da fềmea maior, usando-se um meio de solução salina a $0,9 \%$ heparinizada. O sangue completo foi examinado imediatamente para determinar hematócrito, concentração de hemoglobina e concentrações de ATP e DPG. A concentração de hemoglobina foi determinada agregando 0,02 $\mathrm{ml}$ de sangue a $5 \mathrm{ml}$ de água gelada deionizada, agitando até ficar límpida e medindo a absorbância a 540 e $577 \mathrm{~nm}$. Os coeficientes de extinção milimolares para oxiemoglobina a estas longitudes de onda foram assumidos como sendo iguais aos da $\mathrm{HbA}, 13,8$ e 14,6. respectivamente. Foram usados estojos da Sigma Chemical Co. para a determinação ultravioleta de NTP e a determinação colorimétrica enzímática de DRG.

Os glóbulos vermelhos foram lavados três vezes em solução salina $0,9 \%$ e congelados imediatamente ou após saturação com $\mathrm{CO}$. As células congeladas saturadas com $\mathrm{CO}$ foram levadas a Aarhus, Dinamarca, onde foram determinadas a dependência de temperatura e a afinidade do DPG de oxigênio da hemoglobina. Células congeladas oxigenadas foram levadas a Beaufort, Carolina do Norte onde foram fejtos outros estudos funcionais.

Os glóbulos vermelhos lavados foram lisados com trểs volumes de água fria deionizada e centrifugados para extrair a hemoglodina. A hemoglobina foi purificada usando processos ligeiramente diferentes nos dois laboratórios como é descrito a seguir; uma boa correlação dos cados indica que estas diferenças não influenciaram significativamente as propriedades funcionais da hemoblobina. Em Aarhus a solução de hemoglobina foi dializada em tampão Tris $0,01 \mathrm{M}$ contendo EDTA $5 \times 10^{-4} \mathrm{M}$ e logo libertada de anions, especialmente fosfatos, por passagem através duma resina de intercâmbio iônico de camada mista. A hemoglobina em solução foi concentrada com concentradores B-15 (Amicon, Oosterhut, Holanda). Em Beanfort o hemolisado foi tratado com 0,2 volumes de sulfato de amônio saturado para precipitar os estromas-antes de centrifugar. A solução de hemoglobina foi dialisada em Tris $10 \mathrm{M}\left(\mathrm{pH}\right.$ 9) e EDTA $5 \times 10^{-5} \mathrm{M}$. Em todos os experimentos, o $\mathrm{pH}$ foi variado por adição de Tampão Tris $/ \mathrm{HCl}$ ou bis-Tris $/ \mathrm{HCl}$ dando uma força iônica final de 0,05 . A dependência de temperatura e de DPG da afinidade de oxigênio foram determinadas por equilibrio de $\mathrm{O}_{2}$ usando um método modificado de câmara de difusão de gás. (Nielsel \& Thews, 1961; Weber et al., 1976). A concentração de hemoglobina foi entre 0,30 e $0,38 \mathrm{mM}$ de tetramero nestes experimentos.

$\mathrm{O}$ efeito do $\mathrm{pH}$ sobre a afinidade de $\mathrm{O}_{2}$ em presença e ausência de DPG (100 vezes de excesso sobre o tetramero) ou a um $\mathrm{pCO}_{2}$ de 40 torr foi determinado por um método tonométrico (Riggs \& Wolbach, 1956) usando um espectofotômetro gravador Cary 14. A concentração de hemoglobina foi entre 16 e $20 \mathrm{mM}$ (tetramero). Todos os experimentos foram feitos usando tampōes Tris ou bis-Tris de força iônica constante, I $=0,05$, a temperatura de $20^{\circ} \mathrm{C}$.

As medições cinéticas de dissociação de $\mathrm{O}_{2}$ foram determinadas por mistura rápida como o descrito por Gibson \& Milnes (1964) utilizando um aparelho de "stopped flow" Gibson-Durrum. As medições cinéticas de "fotólise flash photolysis" de combinação de $\mathrm{CO}$ foram determinadas com a aparelho descrito por Bonaventura et al. (1974). A coleção de dados e a análise para todos os experimentos foram feitas, utilizando-se um conversor analítico a digital (Aminco DASAR) conectado a um computador PPD-11 (Digital Equipament Corporation). 


\section{RESULtados}

Os padrões eletroféticos das hemoglobinas dos dois peixes-bois foram idênticos, com uma faixa maior e uma menor $(<5 \%)$. As duas apresentaram maior motilidade eletroforética que a $\mathrm{HbA}$, sendo a faixa menor a mais rápida e tendo assim o ponto isoelétrico menor. $O$ pequeno tamanho de nossa amostra de sangue impediu o fracionamento dos componentes para estudos funcionais individuais. É lamentável que sendo já o final da expediçấo, não foi possível determinar afinidade de oxigênio do sangue completo e efeito Bohr em virtude de ter acabado a provisão de gás.

O hematócrito foi $43 \%$ e a concentração de hemoglobina no sangue completo foi de $2,11 \mathrm{mM}$ (tetramero). A capacidade de oxigênio calculada como $40_{2}$ /tetrâmero é de $18,9 \mathrm{ml}$ $\mathrm{O}_{2} / 100 \mathrm{ml}$. sangue.

A concentração de ATP no sangue completo foi de $0,11 \mathrm{mM}$, o que corresponde a uma taxa DPG/tetramero de 0,33 .

$O$ efeito do DPG sobre o $P_{50}$ e sobre o coeficiente $n$ de Hill para hemoglobina fracionada de peixe-boi está indicado na figura 1 . Sob condições experimentais o DPG diminui marcadamente a afinidade de oxigênio sendo que o efeito depende da concentração.

É notório o fato de que a temperatura corpórea é também baixa para a sspécie amazônica. Medições de temperatura rectal tomadas anteriormente em um Trichechus inunguis de $20,9 \mathrm{~kg}$ deram um valor meio de $33,0 \pm 1,0^{\circ} \mathrm{C}$ $(n=7)$. Uma baixa temperatura corporal está certamente relacionada com a baixa taxa metabólica (como documentado para o manati da Florida por Scholander \& Irving, 1941) e levou a nossa pesquisa de sensitividade a temperatura da ligação de $\mathrm{O}_{2}$.

A figura 2 apresenta a dependência de $\mathrm{pH}$ de $P_{50}$ e $n$ para hemoglobina fracionada de peixe-boi a três temperaturas diferentes. Ainda se a temperatura não parece alterar significativamente o valor de $n$ para esta hemoglobina, o $P_{50}$ aumenta com a temperatura, $\Delta$ log $\mathrm{P}_{50} / \Delta \mathrm{T}=0,025$. A pH 7,4 o valor de $\Delta H$ (calculado como 2,303 RT $\Delta \log \mathrm{P}_{50} /(\Delta-)$, Wyman, 1948) é de aproximadamente $10,2 \mathrm{Kcal}$ $\mathrm{mol}^{-1}$ (incluindo o calor da solução de oxigênio) na faixa de $30-40^{\circ} \mathrm{C}$.

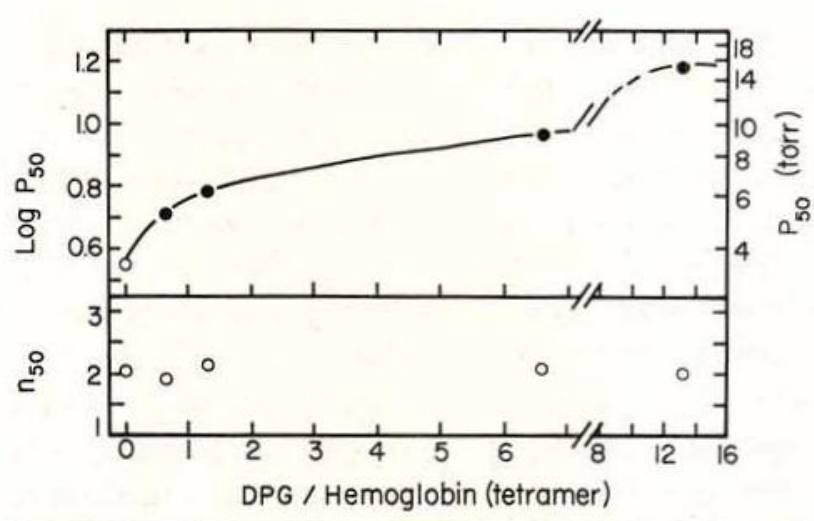

Fig. 1 - Efeito do DPG sobre valores de $P_{50}$ e $\mathbf{n}$ de hemoglobinas fracionadas de Trichechus inunguis, medidos a $30^{\circ} \mathrm{C}, \mathrm{dH} 7,3$, em tampão Tris, força iônica 0,05 . A concentração de hemoglobina é de aproximadamente $0,3 \mathrm{mM}$ (tetrâmero).

$\mathrm{O}$ efeito do $\mathrm{pH}$ sobre a ligação de oxigênio pela $\mathrm{Hb}$ de peixe-boi sob diversas condições está ilustrado nas Fig. 2 e 3 . O efeito Bohr para a hemoglobina fracionada, $\Delta \log \mathrm{P}_{50} / \Delta \mathrm{pH}$ é igual a $-0,4$ a $20^{\circ}(I=0,05)$, mas diminui ligeiramente com aumento na temperatura (Fig. 2). O DPG claramente aumenta o $P_{50}$ e o efeito Bohr através da faixa fisiológica de $\mathrm{pH}$ (Fig. 3). Abaixo de $\mathrm{pH} 7,0$, o DPG diminui ligeiramente o coeficiente $n$ de Hill, indicando cooperatividade reduzida entre as subunidades (Figs. 4A e B). $\mathrm{O} \mathrm{CO}_{2}$ apresenta um efeito inverso sobre o efeito Bohr, diminuindo grandemente a sensitividade a $\mathrm{pH}$ desta hemoglo-

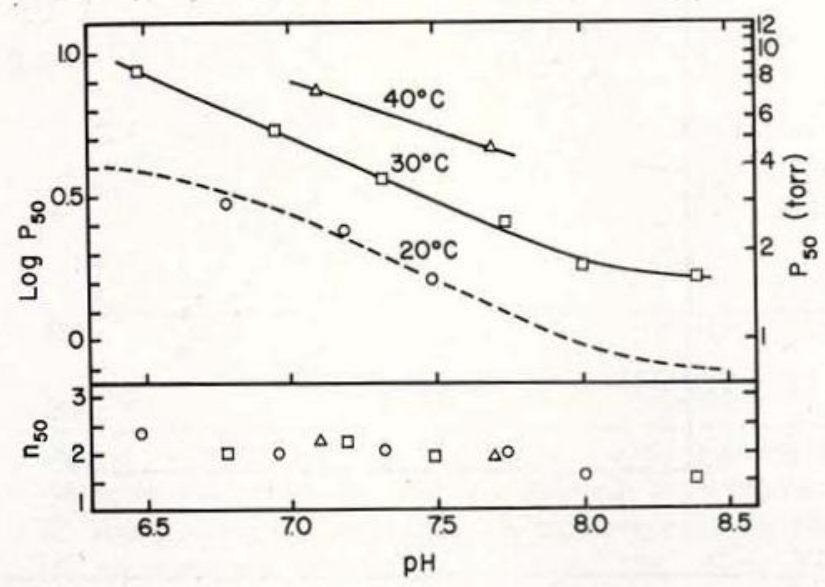

Fig. 2 - Efeito do $\mathrm{pH}$ e temperatura sobre valores $\mathrm{P}_{50} \mathrm{e}$ $n$ de hemoglobina fracionada de $\mathbf{I}$. inunguis medidas em presença de tampões Tris e bis_Tris, $I=0,05$. A concentração de $\mathrm{Hb}$ é $0,38 \mathrm{mM}$ (tetrâmetro) $\Delta 40^{\circ} \mathrm{C} ; \square$, $30^{\circ} \mathrm{C} ; \mathrm{O}, 20^{\circ} \mathrm{C} ;---$, dados graficados da figura $3 \mathrm{sob}$ condiçōes similares. 
bina sem modificar $n$ da condição fracionada (Fig. 3). Pode ser observado, porém, que $n$ também diminui acima de $\mathrm{pH} 7,8 \mathrm{em}$ todas as três condições. Existe uma assimetria evidente nas curvas de Hill sob todas as condições. Também é aparente que a posição das assintotas deoxi varia com $[\mathrm{H}+]$ e a presença de outros fatores alostéricos.

A dissociação de oxigênio de $\mathrm{Hb}$ de peixeboi foi estudada, usando 0 aparelho de "stopped-flow". A $448 \mathrm{~nm}$ as cinéticas exibem três fases distintas em vez das duas normalmente observadas com $\mathrm{HbA}$. A fase final provavelmente corresponde à mudança espectral que ocorre à medida que os dímeros de hemoglobina são agregados para formar tetrâmęros após a desoxigenação (Bonaventura et al.,

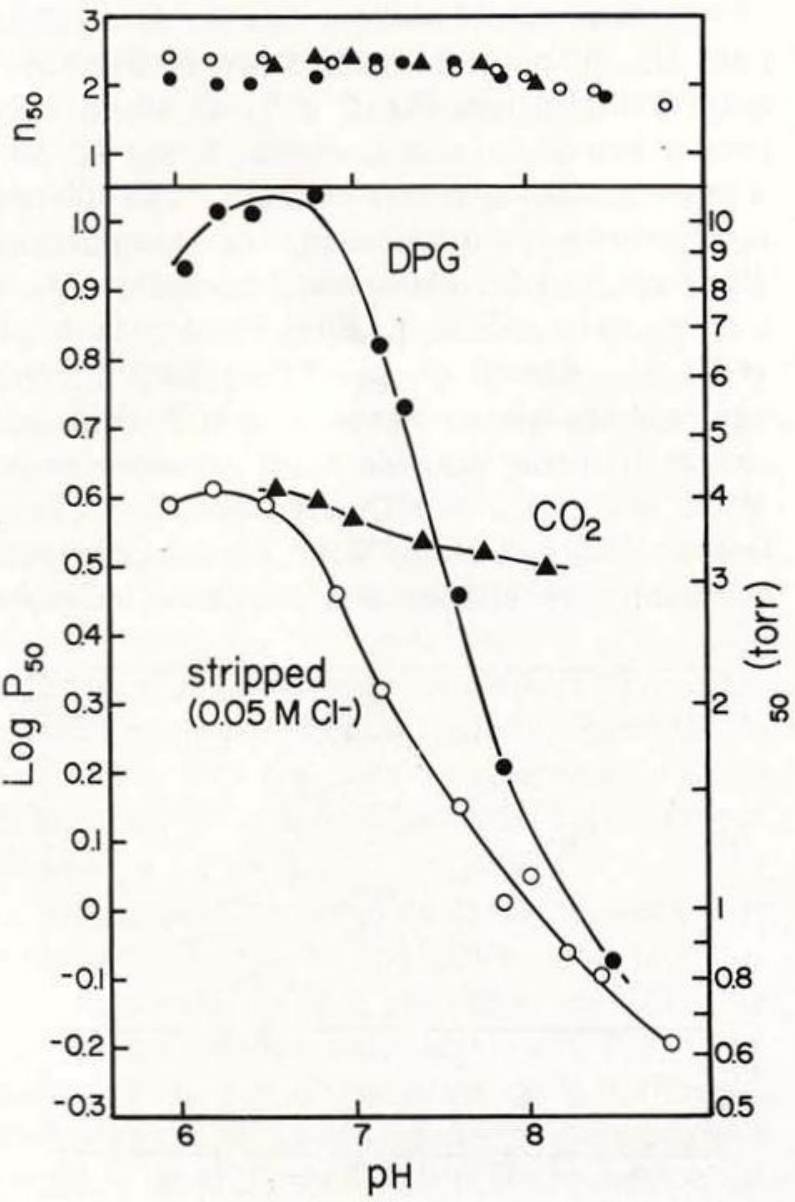

Fig. 3 - Efeito do $\mathrm{pH}$ sobre os valores $\mathrm{P}_{50}$ e $\mathbf{n}$ da $\mathrm{Hb}$ de $I$. inunguis na condiçăo fracionada $e$ na presença de DPG (100 vezes em excesso sobre o tetrâmero) e $\mathrm{CO}_{2}$. Os experimentos de equilibrio foram feitos em presença de tampões Fris e bis-Tris, $1=0,05$ a $20^{\circ} \mathrm{C}$. A concentração de $\mathrm{Hb}$ está entre 0,016 e $020 \mathrm{mM}$ (tetrâmero) $\mathrm{O}$, fracionado do $\mathrm{O}$, DPG; $\mathbf{\Delta} ; \mathrm{CO}_{2}$.

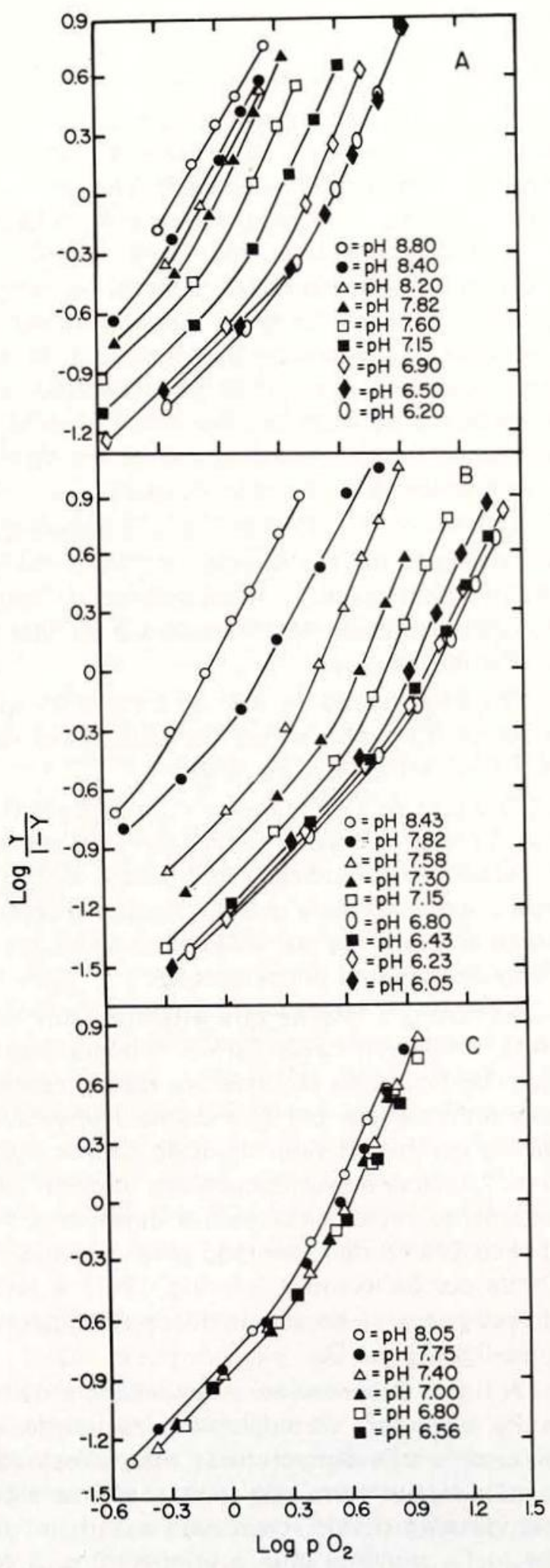

.Fig. 4-Curvas de Hill para ligação de oxigênio de Hb de 1 . inunguis sob as mesmas condiçōes da Fig. 3. A, $\mathrm{Hb}$ fracionado; B, $\mathrm{Hb}$ em presença de DPG (100 vezes em excesso sobre o tetrâmero); $\mathbf{C}, \mathrm{Hb}$ em presença de $\mathrm{CO}_{2}$ (40 torr). 
1974). Esta fase muito vagarosa não é observada a $437,5 \mathrm{~nm}$ (ponto isobestico da fase de agregação) e assim as cinéticas foram seguidas a esta longitude de onda para simplificar a análise. A $437,5 \mathrm{~nm}$, porém, foram observados dois componentes cinéticos chamados fase I e fase II. A pH 7,0 as velocidades das duas fases são $31 \mathrm{seg}^{-1}$ e $6,5 \mathrm{seg}^{-1}$, respectivamente. As duas fases são sensitivas ao $\mathrm{pH}$ e ao DPG, sendo a fase I mais sensitiva que a II.

A velocidade de combinação de $\mathrm{CO}$ cơm a $\mathrm{Hb}$ de peixe-boi também foi medida a 437,5 $\mathrm{nm}$ pela técnica de mistura rápida. Como no caso da cinética de dissociação de $\mathrm{O}_{2}$, as cinéticas de combinação de CO são fortemente dependentes do $\mathrm{pH}$. As cinéticas de combinação de CO também foram examinadas pela técnica de "flash photolysis". Uma velocidade inicial de combinação muito maior foi observada usando este método. Os dois métodos diferem com respeito ao estado inicial da hemoglobina. No aparelho de "stopped flow", a hemoglobina desoxigenada é misturado rapidamente com uma solução tampão equilibrada com $\mathrm{CO}, \mathrm{Hb}+4 \mathrm{CO} \longrightarrow \mathrm{Hb}(\mathrm{CO})_{4}$. Nos experimentos de "flash photolysis" o CO é cau. sado ao dissociar-se da hemoglobina por um "lampejo" intenso de luz e logo recombina, $\mathrm{Hb}(\mathrm{CO})_{4} \rightarrow \mathrm{Hb}+4 \mathrm{CO} \longrightarrow \mathrm{Hb}(\mathrm{CO})_{4}$. No equilíbrio dímero-tetrâmero para $\mathrm{HbA}$, o estado tetrâmero é favorecido na forma não liganda e o estado dímero na forma liganda da hemoglobina. Além disto, os dímeros apresentam uma constante de velocidade de combinação de $\mathrm{CO}$ muito mais alta que os tetrâmeros. Assim, o componente muito rápido de cinética de "flash photolysis", por analogia com HbA, é considerado como representando a combinação dos dímeros com $\mathrm{CO}$. Um gráfico de $\%$ de dímeros a diferentes $\mu \mathrm{M}$ de hemo é apresentado na Figura 5, incluindo um gráfico para $\mathrm{HbA}$ (Bonaventura et al., 1974). Estes dados indicam que na $\mathrm{Hb}$ de peixe-boi a dissociação tetromero-dímero tem uma maior tendência ao estado dímero que em $\mathrm{HbA}$.

\section{DISCUSSÃo}

0 padrão eletroforético da hemoglobina de peixe-boi apresenta uma faixa maior e uma menor. E possível que a faixa menor repre- sente um componente de hemoglobina fetal que poderia diminuir com idade, porém White et al., (1976) encontraram um padrão semeIhante de bandas tanto em adultos quanto indivíduos muito jovens de manatis da Florida Trichechus manatus latirostris. O hematócrito, medido como $43 \%$ é algo menor que o valor de $46,6 \pm 0,5 \%$ obtido por White et al., (1976) para o manati da Florida, mais as medições de concentração de hemoglobina são muito semelhantes para as duas espécies. Tanto a capacidade de $\mathrm{O}_{2}$ quanto $\mathrm{o}$ hematócrito são baixos comparados com os de pinípedos e cetáceos, mas são similares aos de outros mamíferos (Lenfant, 1969; Lenfant et al., 1970). Presumivelmente, a baixa capacidade de $\mathrm{O}_{2}$ está relacionada com os hábitos alimentares e mergulhos rasos do manati. A duração dos mergulhos, porém, não é necessariamente curta. Durante mergulhos mais longos, o notavelmente baixo consumo de oxigênio tenderia a compensar a baixa capacidade de $\mathrm{O}_{2}$ (Scholander \& Irving, 1941).

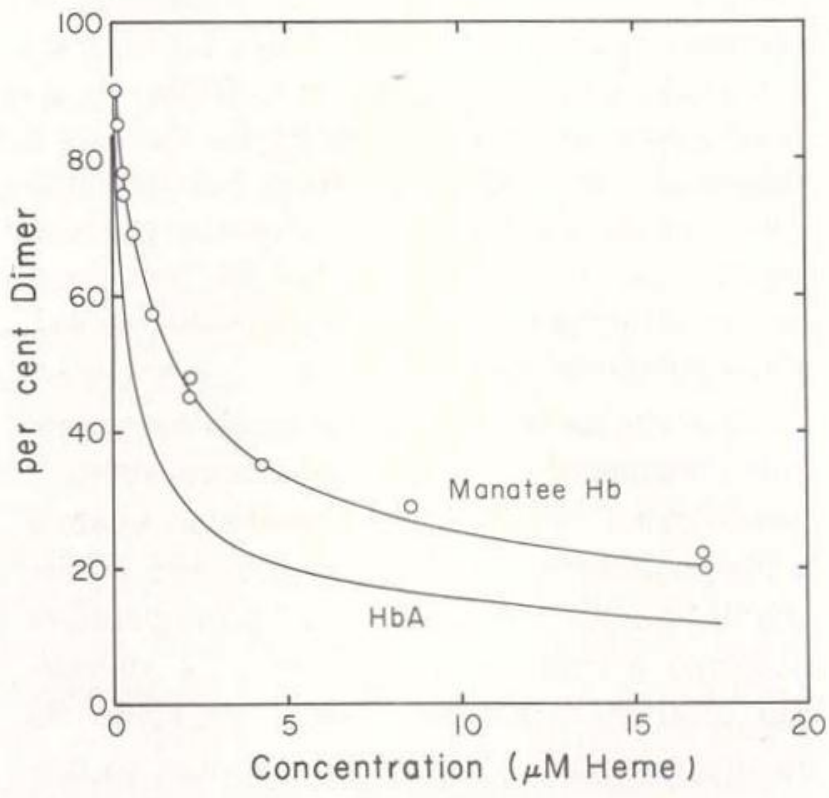

Fig. 5 - Efeito da concentração de proteínas sobre a percentagem de dímeros (determinado em experimentos de flash photolysis em soluçōes carbo-monóxi de $\mathrm{Hb}$ de peixe-boi em tampão bis_.Tris, $1=0,05, \mathrm{pH}=7,0$ $20^{\circ} \mathrm{C}$. A linha de $\mathrm{HbA}$ é para dados obtidos em bisTris $0,05, \mathrm{pH} 7,020^{\circ} \mathrm{C}$ (Bonaventura et al., 1974) : As concentraçōes de C1- são diferentes e podem modificar ligeiramente o equilíbrio de dissociaçăo). A curva ajus. tada que atravessa os pontos dos dados é calculada para um equilíbrio tetrâmero-dímero com constante de dissociaçäo de $0,85 \mu \mathrm{M}$. 
A sensitividade a fosfatos da $\mathrm{Hb}$ de peixeboi é grande ao ser comparada com a de mamíferos onde as concentraçỏes de DPG são baixas (Bunn, 1971); White et al., (1976) reportaram concentrações de ATP e DPG para o manáti da Florida; seus resultados de ATP estão de acordo com os nossos, mas os níveis de DPG deles são muito maiores que os nossos. A seguir, daremos uma explicação plausível para justificar esta discrepância. $O$ estojo de DPG a bordo do "Alpha Helix" foi inadvertidamente congelado contrariando as instruções da Sigma Chemical Co. E possível que o congelamento da fosfoglicerato mutasa resultasse concentrações erroneamente baixas de DPG encontradas em nossos experimentos. Porém baixos níveis de DPG são encontrados em glóbulos vermelhos do gato e de alguns ungulados tais como cabra, boi e ovelha (Harkness et al., 1969; Bunn, 1971) e os baixos níveis de DPG encontrados por nós no peixe-boi amazônico não podem ser ignorados simplesmente por não estar de acordo com os valores anotados por White et al., (1976) para a espécie de Florida. De fato, White et al. (1976) encontraram valores de $P_{50}$ maiores e valores de DPG menores para o manáti muito jovem ao compará-lo com o adulto, o que é o inverso dos valores esperados de prevalecerem as diferenças normais de afinidade de oxigênio materno-fetais.

O efeito da temperatura é muito menor sobre a hemoglobina de peixe-boi que sobre a hemoglobina humana. Sensitividade reduzida à temperatura tem sido reportada para o sangue de um porco-espinho, onde a temperatura do corpo é muito reduzida durante a hibernação (Clausen \& Ersland, 1968). Há evidência de alguma variabilidade na temperatura do corpo do peixe-boi (Dekeyser, 1952; dados não publicados de R.C.B., D.D. \& D.M.), e caso similar tem sido reportado para elefantes (Elder \& Rodgers, 1975). As temperaturas retais do manáti de $33,0 \pm 1,0^{\circ} \mathrm{C}$ foram tomadas em animais em águas com temperaturas variando entre $27-31^{\circ} \mathrm{C}$. Dekeyser (1952) trabaIhando com Trichechus senegalensis em águas variando entre 20 e $22^{\circ} \mathrm{C}$ obteve temperaturas retais de $26 \cdot 6 \pm 0,9^{\circ} \mathrm{C}(n=36)$.
Benesch et al. (1969) encontram para $\mathrm{HbA}$ um $\Delta \log \mathrm{P}_{50} / \Delta \mathrm{T}=0,025$ em Na Cl $0,1 \mathrm{M}$, ou seja, aproximadamente o mesmo valor da $\mathrm{Hb}$ de manáti em um tampão de $\mathrm{I}=0,05$ (Fig. 6) . Na virtual ausência de $\mathrm{Cl}^{-}$, o $\Delta \log \mathrm{P}_{50} / \Delta \mathrm{T}$ é igual 0,07 para $\mathrm{HbA}$ (dados não publicados, C. e J. Bonaventura) . Em tampão de $\mathrm{I}=0,05$, ou seja, o mesmo dos dados de manáti, pode predizer-se que $\mathrm{HbA}$ terá uma sensitividade à temperatura de duas vezes a exibida pela $\mathrm{Hb}$ de manáti (Fig. 6).

Para um animal de sangue quente em um meio aquático, uma sensitividade reduzida da hemoglobina quanto à temperatura pode ser importante para a homeostase. O complexo sistema de intercâmbio de calor dos sirênios (Elsner, 1969) reduziria a perda de calor ao meio externo mas exporia o sangue que passa através dos vasos periféricos a marcadas flutuações de temperatura, especialmente em águas frias. O atum de cauda azul, Thunnus thynnus, mantém uma elevada temperatura de núcleo por um sistema funcionalmente similar de intercâmbio de calor (Carey \& Teal, 1966) e sua hemoglobina e especialmente insensitiva à temperatura (Fig. 6) . O $\Delta \log P_{50} / \Delta T$ de hemoglobina de atum é aproximadamente 0,003

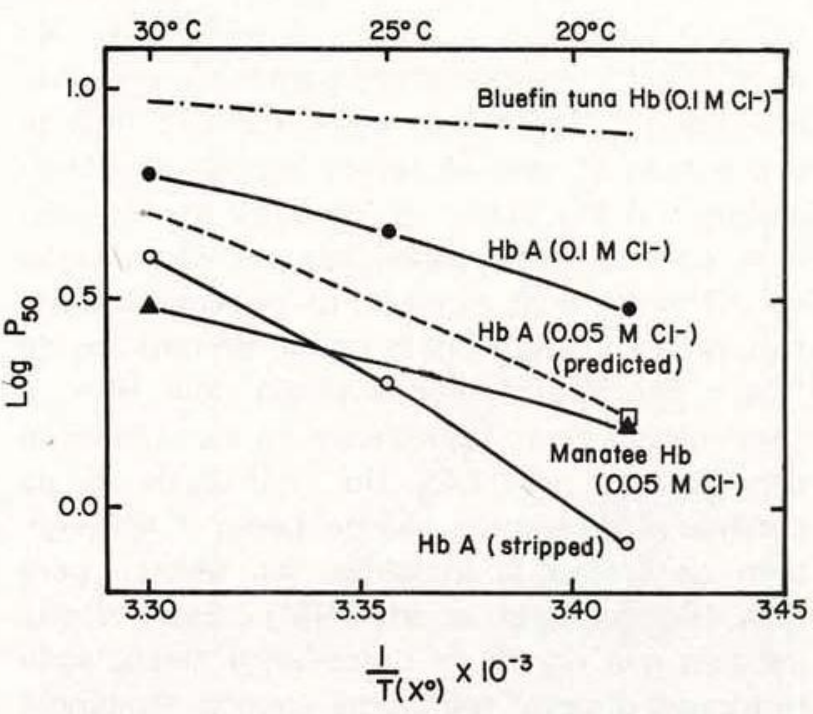

Fig. 6 - Comparação de dependência térmica de várias hemoglobinas sob condições variáveis - . - . - Hb de um atum de cauda azul em C1- 0,1 M (Rossi_Fonelli \& Antonini, 1960); $\bullet-, \mathrm{HbA}$ em C1- $0.1 \mathrm{M}$; 0-0-0, $\mathrm{HbA}$ em ausência virtual de $\mathrm{C} 1-; \square, \mathrm{HbA}$ em $\mathrm{C1} 1-0,05 \mathrm{M}$; em $\mathrm{C}_{1}$ - $0,05 \mathrm{M}$. 
e o $\Delta \mathrm{H}$ é quase zero (Rossi-Fanelli \& Antonini, 1960). Deve anotar-se que este dado foi obtido de hemoglobina cristalina em uma solução de $\mathrm{NaCl} 0,1 \mathrm{M}$ e não 0,05 . Como já foi mencionado para $\mathrm{HbA}$, a diminuição da força iônica aumenta a sensitividade à temperatura assim como a afinidade de $\mathrm{O}_{2}$. Se este fenômeno é generalizado, a sensitividade aparente da $\mathrm{Hb}$ de atum pode estar exagerada ao ser comparada com a $\mathrm{Hb}$ de manáti estudada a $\mathrm{I}=0,05$. Há outros dados quanto a hemoglobinas insensitivas à temperatura de vertebrados poiquilotermos tais como hemoglobinas catódicas de salmão e trutas (Hashimoto et al., 1960; Brunori et al., 1973; Brunori, 1975). Há, por enquanto, pouca informação com relação a sensitividade térmica das hemoglobinas de mamíferos aquáticos ou dos mamíferos com temperaturas corporais variáveis para poder determinar exatamente o significado fisiológico deste fenômeno.

As propriedades cinéticas da hemoglobina de peixe-boi diferem significativamente das de $\mathrm{HbA}$; excluindo a fase muito vagarosa de agregação dímero tetrâmero, a velocidade de dissociação de $\mathrm{O}_{2}$ de $\mathrm{HbA}$ pode ser descrita por um exponencial simples. Em $\mathrm{Hb}$ de peixeboi, a dissociação de $\mathrm{O}_{2}$ é bifásica a $437,5 \mathrm{~nm}$ (ponto isobéstico da fase de agregação). Bonaventura et al. (1974) também observaram bifasicidade nas cinéticas de dissociação das $\mathrm{Hb}$ de Lemur e concluiram a partir desta e outras evidências que as cadeias $\propto$ e $\beta$ da molécula tetrâmera foram responsáveis pelas fases vagarosas (II) e rápidas (I), respectivamente do processo de desoxigenação. Porém, a $\mathrm{Hb}$ de manati difere em vários aspectos da $\mathrm{Hb}$ de Lemur. A fase II da $\mathrm{Hb}$ de Lemur é independente do $\mathrm{pH}$ e essencialmente não é atingida pelo DGP; a fase I é fortemente dependente do $\mathrm{pH}$ e o efeito do $\mathrm{pH}$ é aumentado por DPG. A Hb do peixe-boi apresenta uma dependência de $\mathrm{pH}$ aumentada pelo DPG nas duas fases, mas a fase rápida é mais fortemente afetada tanto pelo $\mathrm{pH}$ quanto pelo DPG como $\mathrm{da} \mathrm{Hb}$ de Lemur.

As curvas de Hill para $\mathrm{Hb}$ fracionadas de peixe-boi, $\mathrm{Hb}$ de peixe-boi + DPG e $\mathrm{Hb}$ de peixe-boi $+\mathrm{CO}_{2}$ (Fig. $4 \mathrm{~A}$, B e C, respectivamente) indicam outro desvio do padrão de $\mathrm{HbA}$. Se HbA apresenta cooperatividade mar- cada acima de $10 \%$ de saturação de oxigênio, a curva de Hill para $\mathrm{Hb}$ de pei-boi mantém uma pendente perto da unidade até pelo menos $30 \%$ de saturação. Isto é evidente através da faixa de $\mathrm{pH}$ em presença dos dois fatores alostéricos, DPG e $\mathrm{CO}_{2}$ (com excepção de que os dados de \% baixa de saturação não foram obtidos a $\mathrm{pH}$ alto em $\mathrm{Hb}$ fracionada). Esta propriedade, se interessante em si mesma, é vantajosa pois permite uma tentativa de análise da parte inferior não cooperativa da curva de Hill, usando-se técnicas fotométricas comuns. Tyuma et al. (1973 a) utilizando um método de gravação automático (Imai et al., 1970), demonstraram que a forma da curva de equilíbrio de oxigênio para a hemoglobina de mamíferos depende, de fato, do $\mathrm{pH}$. A técnica deles permite a análise muita exata de curva a níveis de saturação de oxigênio muito baixos ou muito altos. Tomando em conta a dimerização, a concentração baixas de $\mathrm{Hb}$ (Ackers \& Halverson, 1974) é possível calcular exatamente a constante de Adair para hemoglobina humana e determinar a dependência de $\mathrm{pH}$. Os trabaIhos de Tyuma et al (1973 a, b), Imai (1973) e Imai \& Yonetani (1975) têm mostrado dúvidas, quanto à aplicação à hemoglobina do modelo alostérico de dois estados proposto por Monod et al., (1965) .

Não tendo a parte superior da curva de equilíbrio de oxigênio, estamos limitados aqui a discutir os efeitos relativos dos ligantos sobre a primeira constante de Adair, $\mathrm{K}_{1}$ que é a determinante primeira da parte inferior da curva. $\mathrm{O}$ presente trabalho sobre $\mathrm{Hb}$ de peixeboi está essencialmente de acordo com os resultados de Imai e Yonetani (1975) para HbA. Para as duas hemoglobinas a assintota deóxi é fortemente deslocada em direção a uma menor afinidade com diminuição do pH e o DPG, aumentando esta deslocação. Temos incluido as curvas de ligação de $\mathrm{Hb}$ de peixe-boi em presença de $\mathrm{CO}_{2}\left(\mathrm{pCO}_{2}=40\right.$ torr $)$.

De acordo com a extensão do esquema de Adair apresentado por Imai \& Yonetani, (1975) no qual as cadeias $\propto$ e $\beta$ são tratadas como não equivalentes, $K$, é igual a $(K \propto+K \beta) / 2$ onde $\mathrm{K} \propto$ e $\mathrm{K} \beta$ são constantes de associação das cadeias $\propto$ e $\beta$ combinando-se com a primeira molécula de oxigênio. O número de Prótons Bohr liberados no estado $i+h$ de oxigena- 
ção, $\Delta \mathrm{H}_{\mathrm{i}}+$, pode ser calculado a partir de $\mathrm{K}_{\mathrm{i}}$ usando-se a seguinte relação: $\Delta \mathrm{Hi}+=$ a log $\mathrm{Ki}^{\prime} / \mathrm{a} \mathrm{pH}$. Kilmartin et al. (1973) calcularam o valor de dependência de $\mathrm{pH}$ para valina $1 \propto$, histidina $146 \beta$ e grupos Bohr alcalinos desco nhecidos e estas curvas correspondem bem com as curvas de dependência de $\mathrm{pH}$ de $\Delta \mathrm{H}_{1}+$, $\Delta \mathrm{H}_{2}+$ e $\Delta \mathrm{H}_{3}+$ respectivamente como dados por Imai \& Yonetani (1975). Se a valina $1 \propto$ não toma prótons na deoxigenação, como quando há ligação de $\mathrm{CO}_{2}$, a dependência de $\mathrm{pH}$ de $\Delta \mathrm{H}_{1}+$ deve ser eliminada e, por conseguinte, também a dependência de $\mathrm{pH}$ de $\mathrm{K}_{1}$. Os dados na Fig. $4 \mathrm{C}$ indicam que a porção não cooperativa das curvas de Hill é essencialmente insensitiva a $\mathrm{pH}$ na presença de $\mathrm{CO}_{2}$. Continuando com os argumentos de Imai \& Yanetani (1975) isto indicaria que a dependência de $\mathrm{pH}$ de $\mathrm{K}_{1}$ e eliminada por $\mathrm{CO}_{2}$ como predito admitindo que a extrapolação de $\mathrm{HbA}$ a $\mathrm{Hb}$ de peixe-boi é razoável. Pode ver-se claramente na Fig. $4 \mathrm{C}$ que a dependência $\mathrm{pH}$ da porção média das curvas de ligação também é marcadamente reduzida por $\mathrm{CO}_{2}$. Porém, estes dados não são suficientemente extensos para analisar quantitativamente estes efeitos do $\mathrm{CO}_{2}$ sobre as constantes de Adair. A redução do efeito Bohr por $\mathrm{CO}_{2}$ (40 torr) é algo menor para $\mathrm{HbA}$ que para a $\mathrm{Hb}$ de peixe-boi (Siggaard-Andersen et al., 1972 e M. F., resultados não publicados) e o efeito de $\mathrm{CO}_{2}$ sobre as constantes de Adair para $\mathrm{HbA}$ pode ser algo menos drástico.

Ackers et al. (1975) demonstraram que a presença de pequenas quantidades de dímeros afetam notoriamente a forma da curva de oxigenação. As constantes de Adair calculadas de tal curva dependeram grandemente então, da concentração de hemoglobina em soluções diluídas (Ackers \& Halverson, 1974; Ackers et al., 1975; Mills et al., 1976). A curva para a fração de dímeros VS. a concentração hemo (fig. 5) tem sido ajustada bem satisfatoriamente com uma constante de dissociação de $\mathrm{L}=0,85 \mu \mathrm{M}$. Com base nisto, a concentração de dímeros do derivado totalmente ligado de $\mathrm{HbCO}$ deve ser de aproximadamente $11 \%$ a $60 \mu \mathrm{M}$ de hemo, concentração esta usada nos estudos de equilíbrio de $\mathrm{O}_{2}$. Porém, por influência da"HbA, a conformação deóxi tende a estar 106 vezes menos dissociada que a conformação óxi. Como a $\mathrm{Hb}$ de peixe-boi tende a estar constrita na conformação deóxi a uma faixa inusitadamente larga de saturação de oxigênio, é provável que os dímeros contribuam muito pouco para a forma das porções inferiores da curva de equilíbrio de oxigênio, neste caso.

A pH 6,5 ou 6,8 para $\mathrm{Hb}$ de peixe-boi fracionada ou $\mathrm{Hb}+\mathrm{DPG}$ respectivamente, o efeito Bohr alcalino diminui e embaixo destes valores de $\mathrm{pH}$ há uma mudança a efeito Bohr inverso ou ácido. Na presença e ausência de DPG a assíntota inferior de curva de Hill não se desvia mais em nenhuma direção ainda que o $P_{50}$ diminuia ligeiramente (Fig. 4B). Se $K_{1}$ representa a contribuição da valina $1 \propto$ como foi proposto, o efeito Bohr ácido pareceria não estar relacionado com este resíduo. Isto está de acordo com a hipótese de Perutz (1970) do mecanismo do efeito Bohr ácido. Imai (1973) Tyuma et al. (1973 b) e Imai \& Yonetani (1975), todos usando metodologia similar, mostraram dependência de $\mathrm{pH}$ de $\mathrm{K}_{1}$ tanto para $\mathrm{HbA}$ fracionada qunato para $\mathrm{HbA}+\mathrm{DPG}$. Porém, seus experimentos foram restritos à faixa de $\mathrm{pH}$ do efeito Bohr alcalino. Seria interessante ver se $\mathrm{K}_{1}$ da $\mathrm{HbA}$ é independente do $\mathrm{pH}$ na faixa do eefito Bohr ácido.

A presença de assíntotas inferiores diferentes implica a existência de estado $T$ com diferentes afinidades de oxigênio e que a afinidade de oxigênio destes estados depende do número de prótons ligados. Assim, os íns $\mathrm{H}+$ não só afetam a constante aparente alostérica de equilíbrio, L', como predito pelo modelo de Monod-Wyman-Changlaux (1965), mas também o coeficiente não inclusivo de ligação $C=P_{50}, R / P_{50}, T$, isto é a relação do $P_{50}$ do estado $\mathrm{R}$ com a $\mathrm{P}_{50}$ do estado $\mathrm{T}$ aparente. Esta é a modificação do modelo MWC sugerida por Brouwer et al. (1977) para explicar o desvio da assíntota deóxi das curvas de Hill de ligação de oxigênio pela hemocinina de Limulus com aumento na concentração $\mathrm{Cl}^{-}$. Minton \& Imai (1974) propuseram um modelo de três estados para explicar a presença de mais de um estado de afinidade baixa ou $T$. Os dados apresentados aqui para $\mathrm{Hb}$ de peixe-boi também encaixam dentro deste modelo.

A assimetria das curvas de $\mathrm{Hill}$ da $\mathrm{Hb}$ de peixe-boi é semelhante a exibida pelas curvas de Hill tanto de $\mathrm{HbA}+\mathrm{IHP}$ e $\mathrm{Hb}$ fracionada 
de Lemur a pH baixo (Bonaventura et al., 1974). A Hb de peixe-boi, porém, também apresenta curvas de Hill extraordinariamente assimétricas a $\mathrm{pH}$ alto, possivelmente indicando o envolvimento de uma ponte de sal adicional restringindo a transição de baixa a alta afinidade até que a hemoglobina esteja quase até $30 \%$ saturada com $\mathrm{O}_{2}$. Em virtude de sangue venenoso estar usualmente mais saturado que isto, a função fisiológica do aumento em estabilidade pareceria ser pequena, mas pode ser importante, durante mergulhos prolongados. Uma hemoglobina não cooperativa assemelharse-ia à mioglobina sob estas condições e poderia reduzir a taxa de consumo de $\mathrm{O}_{2}$.

A assimetria das curvas de Hill é considerada como uma indicação de heterogeneidade das cadeias $\propto$ e $\beta$ no $\mathrm{Hb}$ do sangue de Lemur e $\mathrm{HbA}+$ IHP (Bonaventura et al., 1974). A natureza bifásica da cinética de dissociação da $\mathrm{Hb}$ de peixe-boi, tomada em conjunto com a assimetria das curvas de Hill (Fig. 4) sugere marcadamente que a cadeia $\propto$ e a cadeia $\beta$ da $\mathrm{Hb}$ de peixe-boi são, de fato, funcionalmente heterogeneas. Tal heterogeneidade das subunidades tem sido demonstrada para uma série de outras hemoglobinas de vertebrados (Gray \& Gibson, 1971; Tan et al., 1973) o que não é surpreendente, tendo em vista a não identidade das seqüências de aminoácídos das cadeias $\propto$ e $\beta$.

\section{RESUMO}

Em resumo, o hematócrito e capacidade de $\mathrm{O}_{2}$ do sangue de Trichechus inunguis são baixos comparados com os de outros mamíferos mergulhadores, ainda que dentro da média para animais terrestres, o que segue de perto a fisiolơgia respiratória total do manáti (Scholander \& Irving, $1941 \mathrm{em}$ Trichechus manatus latirostris). A hemoglobina também é bastante normal em diversos aspectos porém única em outros. A afinidade de $\mathrm{O}_{2}$ seja em hemoglobina fracionada seja em hemoglobina em presença de DPG ou $\mathrm{CO}_{2}$, é bastante similar a de $\mathrm{HbA}$, através de uma ampla faixa de $\mathrm{pH}$. Certâs outras propriedades funcionais de hemoglobina de peixe-boi são, porém, extraordinárias. A sentividade térmica é marcadamente reduzida, o que pode ser correlato com a baixa e aparentemente variável temperatura corporal do manáti. As cinéticas de ligação de $\mathrm{O}_{2}$ são claramente bifásicas e a diferença de $\mathrm{HbA}$ e a heterogeneidade de cadeia mais marcada. A Hb de peixe-boi tende a dissociar-se em dímeros a baixas concentrações mais facilmente que $\mathrm{HbA}$. Nas curvas de ligação de $\mathrm{O}_{2}$ a assíntota do estado $\mathrm{T}$ aparente está fortemente desviada a menor afinidade com diminuição do $\mathrm{pH}$ quando fracionado ou em presença de DPG, mas não em presença de $\mathrm{CO}_{2}$. Assim, o modelo de dois estados de Monod et al. (1965) não é aplicável à hemoglobina de peixe-boi e são sugeridas alternativas (Minton \& Imai, 1974; Brouwer et al., 1977). As curvas de Hill apresentam assimetria excepcional, mantendo a pendente de unidade através da faixa de $\mathrm{pH}$ de 6 - 9 até alcançar $30 \%$ de saturação com $\mathrm{O}_{2}$. A assimetria é provavelmente uma função tanto da heterogeneidade da cadeia quanto de um mecanismo estabilizador adicional, possivelmente outra ponte de sal que restringe a transição alostérica dos estados $T$ a $R$ desta hemoglobina.

\section{Agradecimentos}

Este trabalho foi apoiado pela bolsa da National Science Foundation PCM 75-06451. A Dra. Célia Bonaventura ajudou a fazer e analisar as cinéticas de fluxo parado e de fotólise de flash. Agradecemos aos Drs. Austen Riggs, Marius Brouwer e Célia Bonaventura pelas discussões estimulantes e valiosas. Também agradecemos a colaboração e apoio dos Drs. Warwick Estevam Kerr e Wolfgang Johannes Junk ao projeto Peixe-boi mantido pelo ConseIho Nacional de Desenvolvimento Científico e Tecnológico (CNPq). O Dr. Joseph Bonaventura é pesquisador estabelecido da American Heart Association e agradece as bolsas de $\mathrm{Na}$ tional Science Foundation (BMS 73-01695) e do National Institute of Health (HI-15460).

\section{SUMMARY}

Hematocrit $(43 \%)$ and $\mathrm{O}_{2}$ binding capacity $(18.8 \mathrm{ml}$ $\mathrm{O}_{2} / 100 \mathrm{ml}$ blood) of Trichechus inunguis blood are low compared to the values for other diving mammals but are similar to those for land mammals. Stripped manatee 
$\mathrm{Hb}$ is similar to human $\mathrm{HbA}$ in its sensitivity to $\mathrm{pH}$, 2,3 - diphosphoglycerate and $\mathrm{CO}_{2}$, but less sensitive to temperature and more prone to dissociate into dimers. The unique Hill plots exhibit no cooperativity below $30 \%$ $\mathrm{O}_{2}$-saturation indicating a highly stabilized " $\mathrm{T}$ " or low affinity state(s); such asymmetric Hill plots together with biphasic $\mathrm{O}_{2}$-binding kinetics could mean chain heterogeneity. The $\mathrm{pH}$ dependence of oxygen binding by the apparent " $T$ " state, hemoglobin as seen in the Hill plots, is enhanced by 2,3-diphosphoglycerate but eliminated by $\mathrm{CO}_{2}$.

\section{BIBLIOGRAFIA}

ACKers, G. K. \& Halverson, H. R.

1974 - The linkage between oxyhemoglobin and subunit dissociation in human hemoglobin. Proc. Nat. Acad. Sci. U.S.A., 4312-4316.

ACKers, G. K.; Johnson, M. L.; Mrlls, F. C.;

Halverson, H. R. \& Shapiro, S.

1975 - The linkage oxygenation and subunit dissociation in human hemoglobin. Consequences for the analysis of oxigenation curves. Biochemistry, 14 : 4128-4134.

BeNesch, P. E.; Benesch, R. \& YU, C. I.

1969 - The oxygenation of hemoglobin In the presence of 2,3-diphodphoglycerate. Effect of temperature, $\mathrm{pH}$ ionic strenght, and hemoglobin concentration. Biochemistry, 8 : 2567-2571.

Blessing, M. H.

1972 - Studies on the concentration of myoglobin in the sea-cow and porpoise. Comp. Physiol.. $41 \mathrm{~A}$ : $471-480$

Bonaventura, C.; Sullivan, J. B. \& Bonaventura, J.

1974 - Effects of $\mathrm{pH}$ and anions on functional properties of hemoglobin from Lemur fulvus fulvus. J. Biol. Chem., $249:$ 3768-3775.

Brouwer, M.; Bonaventura, C. \& Bonaventura, J.

1977 - Oxygen binding by Limulus polyphemus hemocyanin: allosteric modulation by chlorl. de ions. Biochemistry (in press).

1975 - Molecular adaptations to physlological requirements: the hemoglobin system of trout. Current Topics in Cellular Regulation, 9: 1.30 .

BRUnori, M.; Bonaventura, J.; Bonaventura, C.;

GIARdna, B.; Bossa, J. \& ANTONINI, E.

1973 - Hemoglobin from trout: structural and functional properties. Mol. Cell Biochem., 1: 189-196.

BUNN, H. F.

1971 - Differences In the interaction of 2,3-diphosphoglycerate with certain mammallan hemoglobins. Science, 172 : 1049-1050.
Carey, F. G. \& Teal, J. M.

1966 - Heat conservation in tuna fish. muscle. Proc. Natl. Acad. Sci., U.S., 56 : 1464-1469.

Clausen, G. \& Ersland, A.

1968 - The respiratory properties of the blood of the hibernating hedgehog Erinaceus europaeus L. Resp. Physiol , $5:$ 221-233. peus L. Resp. Physiol., 5 : 221_233.

DEKEYSER, P. L.

1952 - Note sommaire sur la temperature rectale du lamantin (Trichechus senegalensis Link) Bull. Mus. Natnl. Hist. Natur. (24(3) : 243-246.

ELDER, W. H. \& RODGers, D. H.

1975 - Body temperature in the African alephant. as related to ambient temperature. Mammo. lia, $39: 395-399$.

ELSNER, R.

1968 - The Biology of Marine Mammals (1969), p. 103.

1969 - Cardiovascular adjustaments to diving. The Biology of Marine Mammals., p. 117-145.

FyHN, H. J.; FYhN, U. E. H.; DAVIS, B. J.;

GARLIK, R. L. \& POWERS, D A.

1978 - Multiple components and polymorphism in Amazonian Fishes. Comp. Biochem. Physiol. (no prelo).

Gibson, Q. H. \& MILNes, L.

1964 - Apparatus for rapid and sensitive spectrophotometry. Biochem. J., 91: 161171.

GRAY, R. D. \& GIBSON, Q. H.

1971 - The bindig of carbono monoxlde to and chains in tetrameric mammalian hemoglobin. J. Biol. Chem., 246 : 5176-5178.

Harkness, D. R.; Ponce, J. \& Grayson, V.

1969 - A comparative study of the phosphoglyceric acid in mammalian erythrocytes. Comp. Biochem. Physiol., 28: 129-139.

Hashimoto, K.; Yamaguch, Y. \& MatsuUra, F.

1960 - Comparative studies of two hemoglobin of salmon-IV. Oxygen dissociation curve. Bull. Jap. Soc. Sci. Fish., $26: 827.833$.

IMAI, $\mathrm{K}$,

1973 - Analysis of the oxygen equilibrium of native and chemically modified human adult hemoglobins on the basis of Adair's stepwise oxygenation theory and the allosteric model of Monod, Wyman and Changeaux. Biochemistry, 12 : 798-808.

IMAI, K.; MARIMOTO, H.; KotANI, M.; WATARI, H.;

HTRATA, W. \& KURODA, M.

1970 - Studies on the function of abnormal hemoglobins. I. An improved method for automatic measurement of the oxygen equi. librium curve of hemoglobin. Biochem. Biophys. Acta., 200: 189-796. 
ImaI, K. \& YotenaI, T.

$1975-\mathrm{pH}$ dependence of the Adair constants of human hemoglobin. J. Biol. Chem., 250: 2227-2231.

Kilmartin, J. V.; Breen, J. J.; Roberts, G. C. K. \& Ho, C.

1973 - Direct measurement of the pk values of alkaline Bohr groups in human hemoglobins. Proc. Natl. Acad. Scl. U.S.A., 70: 1246-1249.

LENFANT, C.

1969 - Physlological propertles of blood of marine mammls. In. The Biology of Marine Mammals, pp. 96-116.

Lenfant. C.: Johansen, K. \& Torrance. J. D.

1970 - Gas transport and oxygen storage capacity In some pinnipeds and the sea otter. Resp. Physiol., 9 : 277.286.

MILls, F. C.; Johnson, M. L. \& Ackers, G. K. 1976 - Oxygenation-linked subunit interations in human hemoglobin: experimental studies on the concentration dependence of oxygenation curves. Biochemistry, 15: 5350 5362.

Minton, A. P. \& AMAI, K.

1974 - The three-state model: a minimal de. scription of homotropic and heterotropic effects in the binding of ligands to hemoglobin. Proc. Natl. Acad. Sci. U.S.A., 71: 1418-1421.

Monod, J.; Kyman, J. \& Changeaux, J. P.

1965 - On the nature of allosteric transitions : a plausible model. J. Mol. Biol., 12 88-118.

Niesel, W. \& Thews, G.

1961 - Ein neues verfahren zur schnellen und genauen Aufnahme der Sauerstoff bindoungskurve des Blutes und konzentrierter $\mathrm{Ha}$ moproteidlosungen. Pflugers Arch. gee. Physiol., 273 : 380-395.

Perutz, M. F

1970 - Stereochemistry of cooperative effects in haemoglobin. Nature, 228 : 726-739

Prosser, C. L.

1973 - Comparative Animal Physiology, 3rd ed. $182 \mathrm{p}$.

Riggs, A. \& Wolbach, R.

1956 - Sulfhydryl groups and the structure of hemoglobin. J. Gen. Physiol., 39 : 585.605.
ROMER, A. S.

1966 - Vertebrate Paleontology, 3rd. ed. 247 p.

Rossi-FANELli, A. \& ANTONINI, E

1960 - Oxygen equilibrium of haemoglobin from Thunnus thynnus. Nature, 186: 895-896.

SCHOLANDER, P. F.

1940 - Experimental investigations on the respira-

SCholander, P. J. \& IRVing, L.

1941 - Experimental investigations on the respiration and diving of the Florida manatee. J. Cell. Comp. Physiol., $17: 169.191$.

Siggard_ANDERSEN, O.; SAlling, N.; NorgaArdPedersen, B. \& RoRTH, M.

1972 - Oxygen-linked hydrogen lon binding of human hemoglobin. Effects of carbon dioxide and 2,3-diphosphoglycerate. III Comparison of the Bohr effect and the Haldane effect. Scand. J. Clin. Lab. Inevst., 29: 185-193.

TAN, A. L. \& Noble, R, W

1973 - Conditions restricting allosteric transitions in carp hemoglobin. J. Biol. Chem., 248: 2880-2888.

Truma, I.; Imai, K. \& Shimizu, $\mathrm{K}$.

1973a- Analysis of oxygen equilibrium of hemoglobin and control mechanisms of organic phosphates. Biochemistry, 12: 1491-14984

Tyuma, I.; Kamigawara, Y. \& ImaI, K.

$1973 \mathrm{~b}-\mathrm{pH}$ dependence of the shape of the hemoglobin.oxygen equilibrium curve. Biochim. Biophys. Acta., 317-320.

WeBER, R. E.; LYKKeBoe, G. \& JOHANSEN, K.

1976 - Physiological properties of eel hemoglobln : Hypoxic acclimation, phosphate effects and multiplicity. J. Exp. Biol., $64 \div 75-88$.

WhITE, J. R.; HARKNESS, D. R.; IsAaCKs, R. E.

\& DUfFIELD, D. A.

1976 - Some studles on blood of the Florida manatory function in diving mammals and birds. Hval. Skr. 22: 1-131.

tee, Trihcechus manatus latirostrls. Comp. Biochem. Physiol., 55A, 413.417.

WYMAN, J.

1948 - Heme Proteins. Advan. Protein Chem., 4: 4: 407-531. 